\title{
Motion estimation using adaptive matching and multiscale methods
}

\author{
Stephanus Suryadarma Tandjung, Teddy Surya Gunawan and \\ Chong Man Nang \\ School of Applied Science, Nanyang Technological University \\ Nanyang Avenue, Singapore 639798
}

\begin{abstract}
Past approaches on motion estimation use iterative algorithm to produce dense motion fields, which is modeled by the energy functions. The optimization strategy such as simulated annealing or iterated conditional mode reorganize the motion fields slowly. This paper introduces adaptive block matching and multiscale smoothing as an initial motion fields for bayesian based motion estimation. The Adaptive block matching is a local intensity matching procedure, which gives a unique matching results. The results are smoothed by multiscale smoothing algorithm. This algorithm is based on kalman filter, but the time domain of this filter becomes the scale domain. The result shows that this strategy can give a more global motion fields than the result of single resolution bayesian motion estimation method. This multiscale smoothing algorithm have numerous possibility to enhance the speed as well as strategy to produce better motion fields.
\end{abstract}

Keywords: : multiscale smoothing, block matching, motion estimation, kalman filter, successive elimination algorithm, partial distortion elimination, adaptive matching

\section{INTRODUCTION}

The intention of the motion estimation project is to build a "true" motion field. The "true" means the true motion of the object and it is not only the matching pixels between two frames. However, the algorithm only accepts image intensities as the only source, as a result many researchers add smoothness constraint into their algorithms. Those constraints are expected that the algorithm can come up with the "true" motion vector. Beside smoothness constraints, another constraint is explored such as discontinuity contraint from line fields or edge fields and segmentation constrain from region fields.

The general strategies for motion estimation that many researcher has been trying can be summarized in Fig. 1. Minimum a pair of images is needed by the algorithm to extract the motion information. The first things that can be done to reduce the computational tasks for motion estimation algorithm is to separate the pixels into static and moving pixels. Of course, this part can be omitted. Because motion estimation also can produces zero motion vector. Motion estimation produces motion fields and if the estimation errors is given to occlusion detection, pixels can be labeled as covered pixels or exposed pixels. In this case, the pixels have been labeled as a static or moving. The moving pixels also can be divided as normal motion or a occlusion pixels. ${ }^{1}$ As it has been mentioned above, the constraint can be many types as long as it help the contruction of motion field. In this paper a multiscale smoothing to shape the motion vector is introduced.

The steps to reach motion estimation objective are divided into several stages. The first stage is to provide motion fields as suitable as possible for the second stages. This first stage is critical, because the improper initial will produce the inaccurate final motion fields. Especially the fast minimization procedure such as Iterated Conditional Mode (ICM), the solutions will be trapped in local minima. The second stage is bayesian framework. The minimization energy can be a type of stochastic processes. ${ }^{2}$ The first stage can be the result of optical flow

Correspondence: Stephanus Suryadarma Tandjung: E-mail: steph@ieee.org;

WWW: http://sentosa.sas.ntu.edu.sg:8000/〜 steph

Teddy Surya Gunawan: E-mail: teddy@sentosa.sas.ntu.edu.sg; Chong Man Nang: E-mail: cmn@sentosa.sas.ntu.edu.sg 


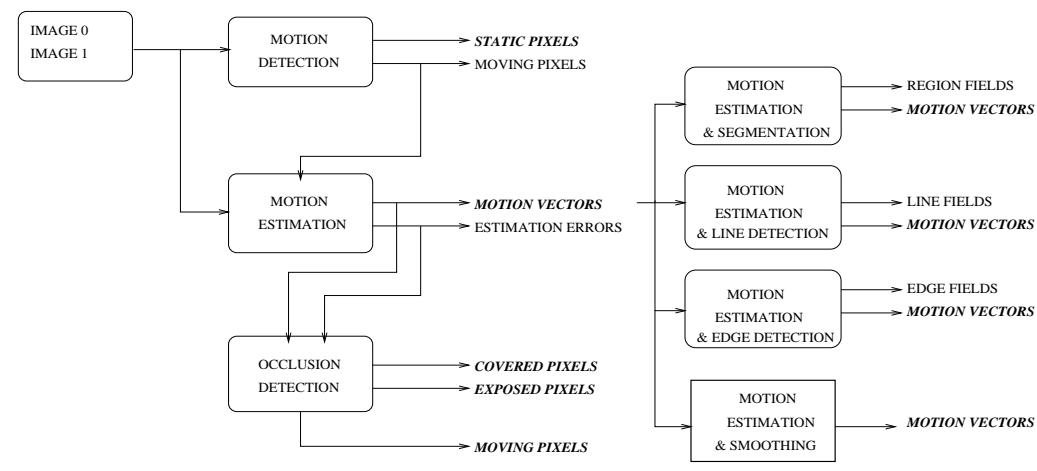

Figure 1. General strategies for motion estimation. The flow process can be arranged in many different ways according to the purpose and algorithms availability.

based motion estimation ${ }^{3}$ or block matching or even no initialization. ${ }^{4}$ But the minimization process will take longer time. The fast and simple initialization is block matching. If a good and fast matching can be presented, the second stage is only the refinement processes.

The commonly used algorithm for motion estimation is block matching algorithm (BMA). The search strategy that will not loose any proposed motion vectors availabe in the searching space is exhaustive search algorithm (ESA). The computational cost for this algorithm is high and it can be reduced by mathematical inequality equation. The algorithm for this fast motion estimation is named successive elimination algorithm (SEA) by Li and Salari. ${ }^{5}$ The speedup is approximately 8 times and another further computational reductions can be achieved appoximately 29 times by inserting partial distortion elimination (PDE), the name of procedure, which is inserted in sum of absolute difference (SAD) procedure. The combination of these algorithms are named SEA + PDE. ${ }^{6}$ Another strategy to reduce the total of proposed motion vector is a combined SAD and MSE. A lower bound for both error measures is exploited to reduce the number of search positions and therefore the computational requirements. ${ }^{7}$

Those algorithms use fix block size. In many cases, the fix block as a reference is not good enough for matching purpose. For example, the less texture reference can match in several proposed motion vector. By increasing the block size and controlling the acceptance of the proposed motion vector that match only on one motion vector, the unique motion vector with the specific block size can be extracted. ${ }^{8}$ The algorithm is called adaptive block size observation model, which is referred to the MRF modeling framework. This problem can be solved by the using of affine transform or higher order motion models. In this paper the simple translation model is used. The result of this algorithm is highly match on pixel intensities. The smoothing processes are needed to adjust the motion fields.

The smoothing processes on the second stage as has been mentioned before are usually done in iterative basis. Another smoothing process is multiscale modeling, ${ }^{9}$ this framework has been used to regularize optical flow computation. ${ }^{10}$ This characteristic of multiscale framework can increase the speed of smoothing process as well as improve the solutions. This paper analysis this possibilities with different types of matching. If the best matching and smoothed motion fields can very fast be developed, the second stage is only a fast refinement process. And the iterative second stage still can be used as well.

\section{TECHNIQUES}

This section introduces the key techniques that are used in the experiments. The fast block matching SEA+PDE is introduced here. This strategy reduces a lot of computation tasks. Adaptive matching, which is currently has a high computation task is introduced subsequently in this section. Although this matching is not well defined yet, because of the high computation tasks, but the strategy to accept only the motion vector, which has a unique minimum error can be put into consideration. Then the multiscale smoothing algorithm from the control 
engineering society that based on kalman filter is also introduced. This algorithm likely becomes a good smoothing strategy to the task to achieve a faster and better "true" motion vectors.

\subsection{Block Matching Algorithm}

Block matching algorithm estimates the motion vector on a block-by-block basis. In this paper, we reviewed and compared various algorithms used to implement block matching algorithm, such as bidirectional motion estimation $(\mathrm{BME}),{ }^{11}$ hierarchical motion estimation $(\mathrm{HME}),{ }^{2}$ and $\mathrm{SEA}^{5}+\mathrm{PDE}{ }^{6}$ To achieve an optimal prediction error, the full search algorithm is used. Moreover, to further reduce the prediction error, the half pixel search is implemented.

The SEA and PDE techniques has no convex assumption for the function of displacement motion vectors. SEA takes the advantage of mathematical inequality. Suppose to find the motion vector for a specific block, we compute maximum absolute difference (MAD) from $N \times N$ block,

$$
\left|\sum_{i=1}^{N} \sum_{j=1}^{N}\right| f(i, j, t)\left|-\sum_{i=1}^{N} \sum_{j=1}^{N}\right| f(i-x,, j-y, t-1)|| \leq \sum_{i=1}^{N} \sum_{j=1}^{N}|f(i, j, t)-f(i-x, j-y, t-1)|
$$

where $f$ is the intensity pixels in position $(i, j)$ and time $t$. The first sum in 1 represents the sum norm of the reference block, and the second sum represents the sum norm of any matching candidate block with motion vector $(x, y)$. The sum norm is computed earlier before the minimization procedure. In every block that will be given a matched motion vector, the comparison is done with the using of sum norm. If the left side of equation 1 is smaller than the previous minimum error, the algorithm must compute the right side of equation 1 to get the correct MAD. The computation reductions can be achieve through the use of checking the accumulate sum row by row on the third sum. If on the specific row the sum is higher than the minimum error, the computation can be stopped. This approach is called partial distortion elimination (PDE) ${ }^{6}$

The comparison of these motion estimation algorithms will be presented in section 4 . Furthermore, we derived new performance index value to fairly assess the algorithm's performances.

\subsection{Adaptive Matching Algorithm}

A suitable initialization motion field can reduce the computational cost needed by the stochastic process in MRF based motion estimation. The initialization process is commonly based on the intensity assumption, known as the implicit constraint. An objective in this model is to add the implicit constraint into the observation model. This model yields a motion vector that has been tested as the only best candidate for a given point. Equation 2 is observation model on the left term and a block model on the right term. Both model will have a minimum energy value according to $d$, the displacement vector and $h$, the block size. $\lambda$ is a weighting functions, $g$ is the image, $l$ is line field.

$$
\min _{d, h}\left\{\lambda_{g} U_{g}\left(g_{t} \mid d, l, h, g_{t-1}\right)+\lambda_{h} U_{h}\left(h \mid g_{t-1}\right)\right\}
$$

Equation 3 is the functions of the model.

$$
\min _{d, h}\left\{\lambda_{g} \sum_{x=1}^{h} \frac{\left[g_{t}(x)-g_{t-1}(x+d(x))\right]^{2}}{2 \sigma^{2}}+\lambda_{g} \Theta(h)\right\}
$$

where $h$ denotes the order of MRF neighborhood system and $\sigma^{2}$ denotes the variance of pixel intensities and $x$ is a pixel site. The auxiliary variable for "indiscriminate texture" variable is

$$
\Theta=\left\{\begin{array}{cc}
\Theta+1 ; & U_{g}\left(g_{t} \mid \widehat{d}_{(i, j, k)}, l, h, g_{t-1}\right)- \\
& U_{g}\left(g_{t} \mid \widehat{d}_{(i, j, k-1)}, l, h, g_{t-1}\right)=0 \\
0 ; & \text { else }
\end{array}\right.
$$


This adaptive observation model is for local motion estimation. The algorithm in this paper has a purpose to exploit the implicit information on the beginning of optimization process. The matching based motion estimation is named implicit approach, Then, the proces is continued by the regularization technique (MRF based). In this technique, the explicit information, as prior knowledge is exploited that the matching error should be the minimum on only one motion vector. The aperture problem is solved in this approach and the occlusion locations can be detected.

\subsection{Multiscale Smoothing Algorithm ${ }^{12}$}

The motion fields on the finest resolution can be smoothed with this multiscale smoothing. For block matching, the finest resolution is not per pixel per motion vector but per block per motion vector. It is the one that can be processed by this algorithm. Remember that block matching employes implicit smoothing constraint, in this case, the result can not be smoothed anymore.

The algorithm produces the best estimate of the process given all available measurements. The measurement can consist of different measurements error or no measurement on specific points, which likely can help to reduce the speed of measurement, in this case matching algorithm. E. g. the matching is done on edge and higly texture object only, the rest is no measurement. The multiscale process:

$$
x(s)=A(s) x(s \bar{\gamma})+B(s) w(s)
$$

is the process from finest scale $s$ and propagate to higher scale $s \bar{\gamma}$ in quadtree until the lowest scale. $w(s)$ is a zero-mean unit-variance white noise process that is added to the model with a gain $\beta$. The measurement equations:

$$
y(s)=C(s) x(s)+v(s)
$$

where, $v(s)$ is a zero-mean white noise process with covariance $R(s)$, this covariance can be related to the matching algorithm, the value of its error or the size of block. A further research is needed in this strategy.

To use this algorithm, it must give the states $x(s)$ at the root node: zero mean with covariance $P(0)$. To compute the prior covariances of all states at individual nodes on the tree via Lyapunov equation

$$
P(s)=A(s) P(s \bar{\gamma}) A^{T}(s)+B(s) B^{T}(s)
$$

The core of multiscale algorithm consists of an upward estimation sweep and a downward smoothing sweep. Define the following quantities:

- $Y(s)=\{y(\sigma) \mid \sigma$ is a descendant of $s\}$ is the collection of measurements at all nodes below s but not including $s$.

- $\widehat{x}(\sigma \mid s)=E\left[x(\sigma) \mid \sigma \in Y_{s} \bigcup y(s)\right]$ is the best estimate of $x(s)$ given measurement at node $s$ and all nodes below $s$

- $\widehat{x}(\sigma \mid s+)=E\left[x(\sigma) \mid \sigma \in Y_{s}\right]$ is the best estimate of $x(s)$ given measurement at all nodes below $s$

- $\widetilde{P}(\sigma \mid s)=\operatorname{Cov}[x(\sigma)-\widehat{x}(\sigma \mid s)]$

- $\widetilde{P}(\sigma \mid s+)=\operatorname{Cov}[x(\sigma)-\widehat{x}(\sigma \mid s+)]$

The upward sweep initializes at the finest level from the prior covariances: $\widehat{x}(s \mid s+)=0, P(s \mid s+)=P(s)$ and the upward model

$$
\begin{gathered}
x(s \bar{\gamma})=F(s) x(s)+\bar{w}(s) \\
y(s)=C(s) x(s)+v(s)
\end{gathered}
$$


where $F(s)=P(s \bar{\gamma}) A^{T} P(s)^{-1}, E\left[\bar{w}(s) \bar{w}(s)^{T}\right]=P(s \bar{\gamma})-F(s) A(s) P(s \bar{\gamma})=Q(s)$

The upward sweep computes the best estimate of the state at a node given all measurements at or below that node. It consists of three steps at each scale: The update step:

$$
\begin{gathered}
\widehat{x}(s \mid s)=\widehat{x}(s \mid s+)+K(s)[y(s)-C(s) \widehat{x}(s \mid s+)] \\
P(s \mid s)=[I-K(s) C(s)] P(s \mid s+) \\
K(s)=P(s \mid s+) C^{T}(s)\left[C(s) P(s \mid s+) C^{T}(s)+R(s)\right]^{-1}
\end{gathered}
$$

The updated estimate is the best estimate of $x(s)$ given all measurements at or below s. The prediction step:

$$
\begin{gathered}
\widehat{x}\left(s \mid s \alpha_{i}\right)=F\left(s \alpha_{i}\right) \widehat{x}\left(s \alpha_{i} \mid s \alpha_{i}\right) \\
P\left(s \mid s \alpha_{i}\right)=F\left(s \alpha_{i}\right) P\left(s \alpha_{i} \mid s \alpha_{i}\right) F^{T}\left(s \alpha_{i}\right)+Q\left(s \alpha_{i}\right)
\end{gathered}
$$

The predicted estimate is the best estimate of $x(s)$ given all measurements at node $s \alpha_{i}(i=1 \ldots q)$ or below. The merge step:

$$
\begin{gathered}
\widehat{x}(s \mid s+)=P(s \mid s+) \sum_{i=1}^{q} P^{-1}\left(s \mid s \alpha_{i}\right) \widehat{x}\left(s \mid s \alpha_{i}\right) \\
P(s \mid s+)=\left[(1-q) P(s)^{-1}+\sum_{i=1}^{q} P^{-1}\left(s \mid s \alpha_{i}\right)\right]^{-1}
\end{gathered}
$$

The merge step combines the predicted estimate of $x(s)$ given each of its child subtrees. The merged estimate is the best estimate of $x(s)$ given all measurements below node s.

The downward sweep computes the best estimate of the states at a node given all available measurements everywhere on the tree:

$$
\begin{gathered}
\widehat{x}(s \mid 0)=\widehat{x}(s \mid s)+J(s)[\widehat{x}(s \bar{\gamma} \mid 0)-\widehat{x}(s \bar{\gamma} \mid s) \\
P(s \mid 0)=P(s \mid s)+J(s)[P(s \bar{\gamma} \mid 0)-P(s \bar{\gamma} \mid s) J(s) \\
J(s)=P(s \mid s) F^{T} P^{-1}(s \bar{\gamma} \mid s)
\end{gathered}
$$

The equation 17 is the result, the estimate motion vectors. 


\begin{tabular}{|l||l|}
\hline Video sequence & Characteristic \\
\hline \hline Claire $(352 \times 288 \times 50)$ & slow object translation with low motion activity \\
\hline Salesman $(352 \times 288 \times 50)$ & slow object translation and panning \\
\hline Basketball $(1920 \times 1024 \times 30)$ & general motion picture with high motion activity \\
\hline Flag $(1920 \times 1024 \times 40)$ & object translation \\
\hline
\end{tabular}

Table 1. The test video sequences

\section{MATCHING ALGORITHM-MULTISCALE SMOOTHING INTEGRATION}

Motion estimation can be divided into two parts, the sensor and the process. Later another processes that can be put into the whole process is the intelligence interpretation or any other higher level algorithm. The sensor part in this case can be optical flow result, or block matching result or adaptive matching result. The adaptive matching result can be put into the focus of this paper, because this matching has the ability to enlarge the block size. The enlarging happen on some degree until a motion vector with a unique measurement error value is achieved. This approach can be thinked as a variable size aperture for the sensor. It is also possible to be put on the different resolution on the quadtree of multiscale smoothing algorithm. Meanwhile, all the measurement is put on finest resolution.

After the adaptive matching has been done. In this stage, the most possible matching for the motion fields based on intensity has been achieved, then the smoothing can be done from the result. The strategy to separate matching and smoothing is proposed in this paper. After multiscale smoothing, the result is the motion fields that follow Markov Random Fields modeling in scale.

When the fix block matching is used to supply this multiscale smoothing, the finest resolution cannot be put into the algorithm, but few level from the finest scale according to block size can be supplied. This is because the implicit smoothness already plays in the development of motion fields.

\section{EXPERIMENTAL RESULTS}

The experiment reported here consists of four parts. The first part is a common block matching algorithm with various strategy. From this experiment, researcher can get a glimpse understanding about the characteristic of each sequence, given PSNR, entropy and computation time as variables. Then adaptive matching is shown here, the experiment supports the intention of this strategy, but no real sequence experiment is shown for this strategy. The multiscale smoothing experiment shows the richness of this algorithm and likely further researchs will follow this presentation. The last experiment is the proposed strategy. In this experiment the smoothing tremendously influenced the result of adaptive matching. Additional result is added when bayesian method or iterative method resmooths the result of multiscale smoothing.

\subsection{Comparison of various block matching algorithms}

In this section, we compare various block matching algorithms, such as: block matching motion estimation (ME), bi-directional motion estimation (BME), hierarchical motion estimation (HME), bi-directional hierarchical motion estimation (BHME), and SEA+PDE. The video sequences used in our experiments is shown in Tab. 1 and Fig. 2.

The apparatus for our experiment is a PC with Intel Pentium III $450 \mathrm{MHz}, 512$ Mbytes memory, and Linux operating system. We used $\mathrm{C}$ and $\mathrm{C}++$ language, and we did not use any machine specific code or assembly language. Moreover, we compile the program using the best compiler option as possible :

$\mathrm{g}^{++}$program.cc -o program -03 -mpentiumpro.

We tested several frames for each algorithm, and take the average value of PSNR, entropy, and elapsed time for each sequence. Figure 3, 4, and 5 show the result for the PSNR, entropy, and elapsed time for each video sequence. The minimum, mean, and maximum values are also shown in the figures.

From figure 3, the PSNR of Claire and Salesman sequences are higher compared to Basketball and Flag sequences. Basically, each video sequence has its own characteristics as shown in the Table 1 . The sequence, in 

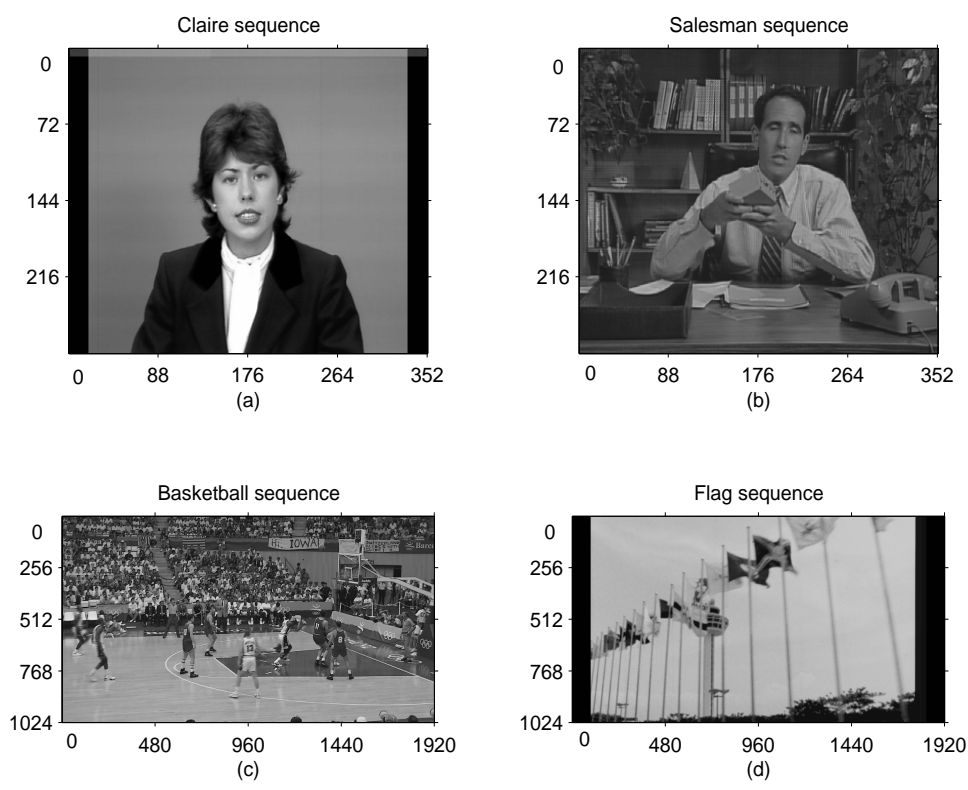

Figure 2. The frame of the test video sequences
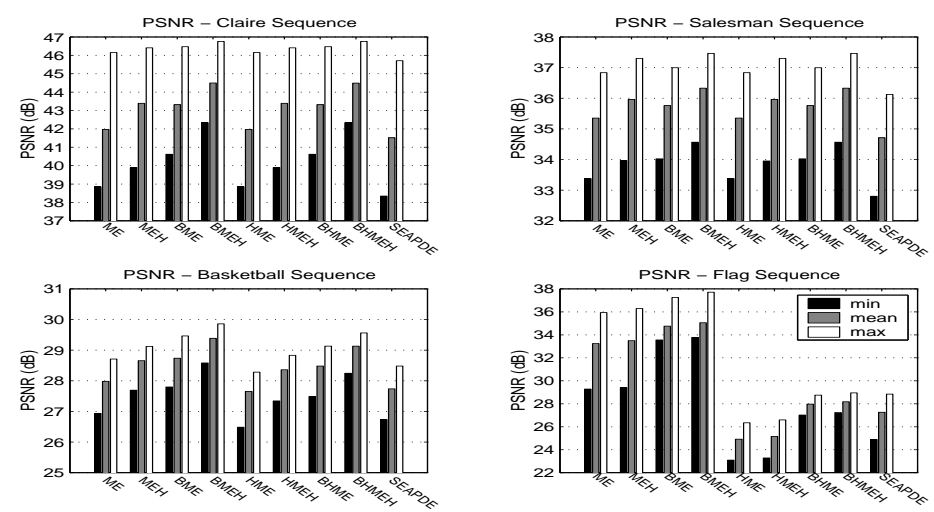

Figure 3. The PSNR result for each video sequences(Note: The SEA should have the same result as ME, however, the padding that is added in SEA+PDE algorithm makes a difference with the rest of experiment).

which low motion activity is dominant, has a high PSNR. To take into account the characteristic of each sequence and to make a fair assessment of PSNR, we derive another standard of assessment as shown in

$$
\delta=\frac{P-P_{0}}{P} \times 100 \%
$$

while $P$ is the PSNR of the reconstructed frame and the current frame, $P_{0}$ is the PSNR of the current frame and the previous frame (Frame Difference), and $\delta$ is the percentage of PSNR improvement, if we are using the result of motion estimation (previous frame + motion vector) instead of using the previous frame alone as a reconstructed frame. Using the equation 20, we can now fairly assess the PSNR result of video sequences and we can explain why one video sequence has a higher PSNR than anothers. Table 2 shows the $\delta$ value for each video sequence and algorithm.

From the table 2, we can see that the Flag sequence has the highest $\delta$ value, in which the motion algorithms can improve the PSNR by 17.48-38.78\%. But we can see also the drawback, by which the entropy value for the Flag sequence is relatively higher compared to others, as shown in Fig. 4. 

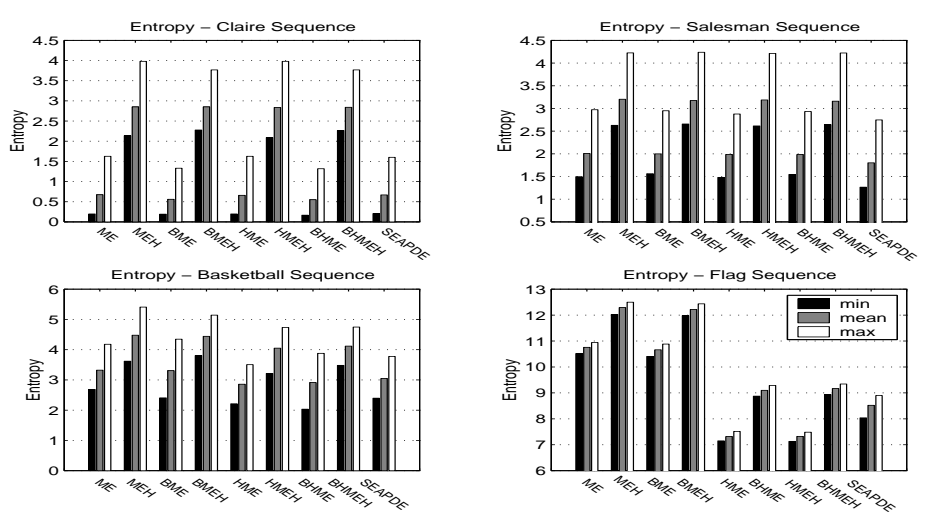

Figure 4. The entropy result for each video sequences (Note: Lower entropy does not mean better result, it is a comparative value that is specified for each sequence)
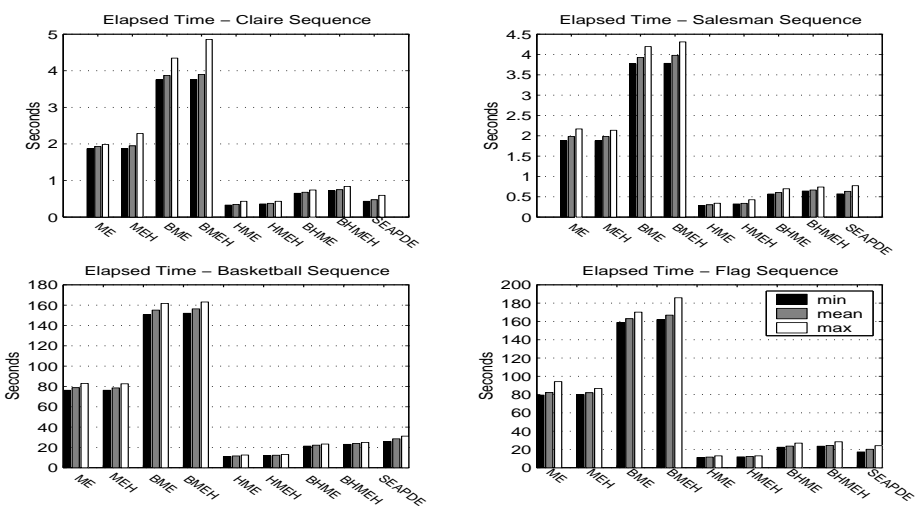

Figure 5. The elapsed time result for each video sequences

In video compression application, one wishes to minimize the entropy for cheaper transmission and to maximize the PSNR for better reconstructed picture quality. ${ }^{13}$ To accomodate the two constraints above, we define the PSNR-to-entropy-ratio as shown below,

$$
\rho=\frac{P}{E}
$$

while $P$ is the PSNR (dB), $E$ is the motion vectors entropy (bits), and $\rho$ is the PSNR-to-entropy-ratio (dB/bits). The higher $\rho$ value means the higher the PSNR (the better picture quality) and the lower the entropy (the smoother the motion vectors, but it is not the measurement of the "true" motion vectors), vice versa.

In order to evaluate which algorithm has the best performance over the others, we derived a performance index by taking into account the PSNR, the entropy, the elapsed time, and the total number of pixel processed. We define the performance index $(Q)$ as,

$$
Q(\rho, t, T)=\frac{\rho \times T}{t}\left(\frac{d B \cdot \text { pixels }}{\text { bits } \cdot \text { seconds }}\right)
$$

while $\rho$ is the PSNR-to-entropy ( $\mathrm{dB} / \mathrm{bits}$ ), $t$ is the elapsed time (seconds), $T$ is the total pixel processed (pixels), and $Q$ is the performance index. Using the equation 22, table 3 shows the $\mathrm{Q}$ value of all the motion algorithms for each sequence. The higher the $Q$ value the better the picture quality, the lower the entropy (the smoother the motion vectors), and the smaller the elapsed time (the faster the process). 


\begin{tabular}{|c|c|c|c|c|c|c|c|c|c|}
\hline & ME & MEH & BME & BMEH & HME & HMEH & BHME & BHMEH & SEAPDE \\
\hline \hline Claire & 6.278 & 9.342 & 9.340 & 11.735 & 6.275 & 9.344 & 9.338 & 11.731 & 5.250 \\
\hline Sales. & 5.676 & 7.270 & 6.762 & 8.221 & 5.673 & 7.268 & 6.763 & 8.223 & 3.928 \\
\hline Basket. & 17.323 & 19.265 & 19.499 & 21.275 & 16.335 & 18.422 & 18.775 & 20.583 & 16.592 \\
\hline Flag & 38.150 & 38.613 & 38.281 & 38.781 & 17.481 & 18.275 & 23.935 & 24.470 & 24.579 \\
\hline
\end{tabular}

Table 2. The $\delta$ value of the video sequences

\begin{tabular}{|c|c|c|c|c|c|c|c|c|c|}
\hline & ME & MEH & BME & BMEH & HME & HMEH & BHME & BHMEH & SEAPDE \\
\hline \hline Claire & 3274087 & 792497 & 2026542 & 405746 & 18672180 & 4110828 & 11752979 & 2115456 & 13228961 \\
\hline Sales & 901763 & 573784 & 461941 & 291760 & 5934919 & 3377073 & 3033091 & 1746642 & 3075495 \\
\hline Basket & 73706 & 65307 & 39336 & 33788 & 573762 & 445169 & 317751 & 248281 & 312129 \\
\hline Flag & 210009 & 160059 & 110183 & 83205 & 1638450 & 1116640 & 865953 & 582939 & 629164 \\
\hline
\end{tabular}

Table 3. The performance comparison of the motion algorithms for each video sequences

From the table 3, we can see that the HME algorithm has the highest $Q$ value. It means that using the $Q$ value, the HME algorithm has the highest picture quality, the smallest entropy (the most compressible motion vector), and the fastest processing time, compared to other motion estimation algorithms. But, we must also take into account the purpose of the finding of motion vectors. If one wants only to achieve the highest PSNR as possible without taking into account the entropy and the elapsed time, then the BMEH algorithm would be the choice. Nevertheless, our experiments show the overall performance for different motion estimation algorithm, in which one can choose which algorithm fit into their needs.

\subsection{Adaptive Matching Algorithm}

This algorithm is likely good for cartoon like sequence, where the flat texture or noiseless sequence exists. To show the matching idea, a synthetic sinus circle is generated as shown in Fig. 6 (a) and (b). Adaptive matching produces fig. 6 (c) in once time running, but the speed is slow enough. The line fields that detect the different between motion vectors are shown in fig. 6 (d) for displaying purpose. They show that several sites have different motion vector. If the threshold of the possible enlargement of block size is reduced, the occlusion model can be neglected as shown in 6 (e). Figure 6 (f) is the difference between first and second images. The black pixels are the sites in which the adaptive algorithm detects as a no motion (zero motion vector). A block of real one slice of image is shifted several pixels away. The fix block size block matching cannot produce a correct motion vectors in once time process (fig. $6(\mathrm{~g})$ ), but adaptive matching can produce the correct motion vectors (fig.6 (h)).

\subsection{Multiscale smoothing}

The model for this multiscale smoothing is: ${ }^{9}$

$$
x(s)=a x(s \bar{\gamma})+4^{-\delta m(t) / 2} w(s)
$$

where $a=1$ and $\delta$ controls the scale-to-scale geometric decay in the variance of the noise term, $w(s)$ and $m(t)$ is the scale. This model has a property that its fine-scale variations have smaller variance than its coarser-scale variations. The starting prior covariance $P=100$. The measurement noise variance $v(s)$ are constant all over the point measurement and $C=1$.

$$
y(s)=C(s) x(s)+v(s)
$$

Given the $16 \times 16$ motion vectors in fig. 7 (a), the smoothing process smooths the motion vector according to the neighbor motion vectors. When the motion vector is rough, the smoothing process adjust the motion vectors as seen in fig 7 (b), the displayed motion vector length is looks closer to the middle motion vector $(4,4)$. This motion vector is smaller than the initial in (a).

Another approach is the difference measurement error on each pixel. The error value (displacement block difference) or the confidence level of the matching algorithm can be related to the measurement noise variance 


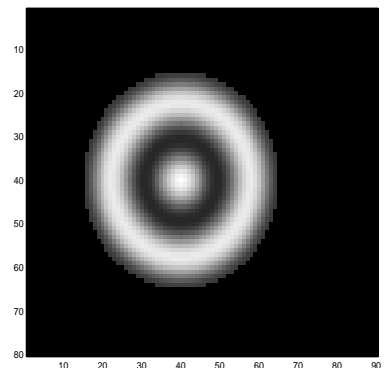

(a) First image

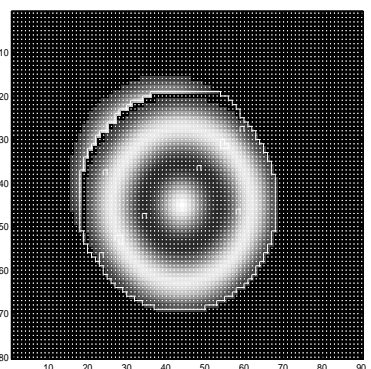

(e) Occlusion sites are excluded

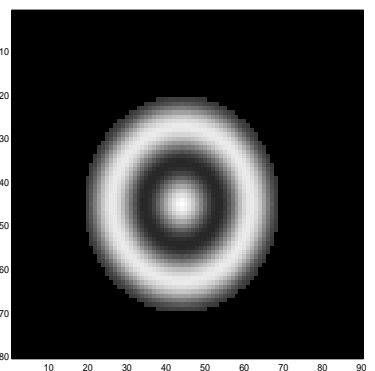

(b) Second image

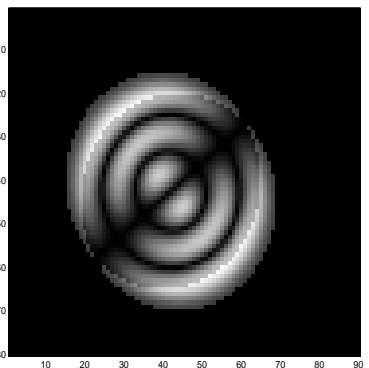

(f) Difference

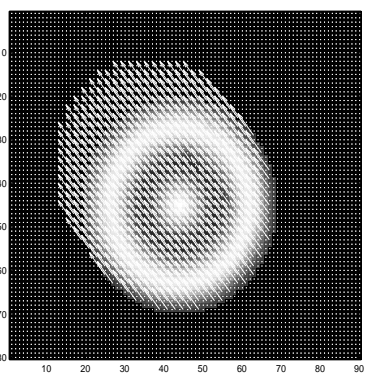

(c) Motion vector

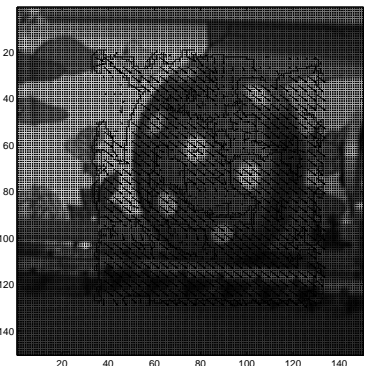

(g) Fix blocksize overlap BM

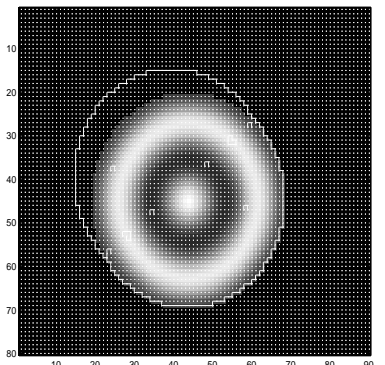

(d) Motion vector with line fields

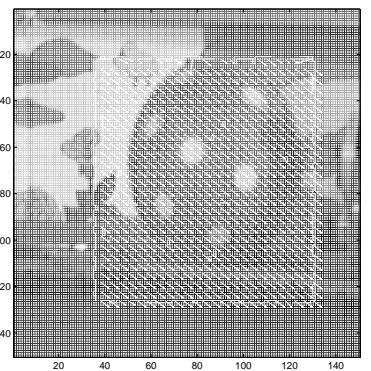

(h) Adaptive block size for matching

Figure 6. Adaptive matching

$v(s)$ to give more information about how much does the multiscale smoothing algorithm can adjust those motion vectors. Fig. 7 (c) shows the different smoothing result because position $(4,4),(3,4),(4,3)),(5,4),(4,5)$ the measurement noise variance is 2 times larger than others. Two upper zero motion vector now becomes same as the mayority neighbor, but the other two lower motion vector remain zero. This condition is caused by the grouping of quadtree. The center $(4,4)$ with longer motion vector, which belongs to upper group affects the two upper zero motion vector. This phenomena is interesting. Based on the measurement error or the confidence level of measurement, the smoothing algorithm can produce sdifferent result.

If there is no measurement available, the smoothing algorithm will receive no measurement information from the matching/sensor and the algorithm will justify the motion according to the surrounding. Fig. 7 (d) is the result when there is no measurement in position $(4,4)$.

With this same smoothing algorithm, given the measurement noise information as well as the motion fields can produce three types of output. In the future research, this phenomena can be used in greater relationship with the matching algorithm. On the next section an equal measurement noise is elaborated.

\subsection{Integration of matching and smoothing}

A small moving part of calendar train sequence is used in this experiment. Note that the motion vector arrows in this paper has the meaning "pixel moves from the pointer end instead of 'to' as is commonly used". The ball is gliding to the left, the background and rail are static but the camera is panning to the left, the train is moving to the left. Adaptive matching produces unsmoothed motion fields and than the smoothing algorithm adjusts the result as seen on fig. 8. Table 4 shows that the PSNR lower and the Entrophy is also lower. For PSNR, it can be understood that smoothing is based on the configuration of motion fields instead of intensity. The entropy value is 


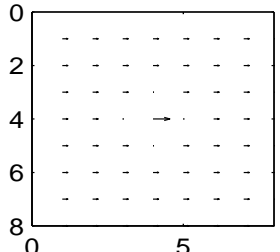

(a)

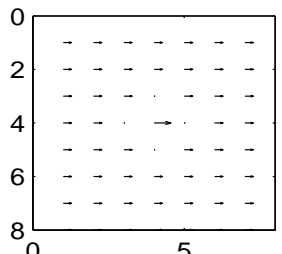

(b)

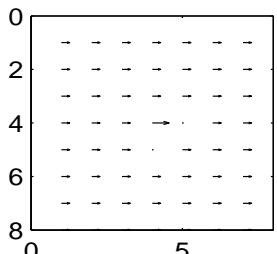

(c)

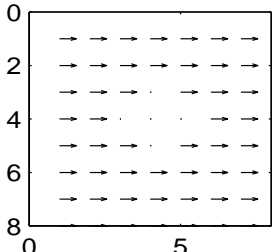

(d)

Figure 7. (a) One motion vector is different with the sorrounding; and (b) all motion vectors are the same (The figure motion vector length is relative in each figure); (c) with different measurement noise variance; (d) with no measurement information.

\begin{tabular}{|c|c|c|c|c|}
\hline Types of process & Figure & PSNR $(\mathrm{dB})$ & Entropy(bits) & $\rho(\mathrm{dB} / \mathrm{bits})$ \\
\hline \hline Adaptive matching & $6(\mathrm{c})$ & 37.66 & 6.20 & 6.07 \\
\hline 4 by 4 block matching & $6(\mathrm{e})$ & 33.55 & 5.54 & 6.06 \\
\hline adaptive matching and multiscale smoothing & $6(\mathrm{~d})$ & 27.79 & 4.97 & 5.59 \\
\hline iterative matching-smoothing (MRF modeling) & $6(\mathrm{f})$ & 37.46 & 3.86 & 9.70 \\
\hline result of (d) is followed by iterative method & $6(\mathrm{~g})$ & 37.37 & 4.24 & 8.81 \\
\hline result of (e) is followed by multiscale smoothing & $6(\mathrm{~h})$ & 29.49 & 4.64 & 6.36 \\
\hline
\end{tabular}

Table 4. The PSNR, Entropy, and $\rho$ of different processes

lower because the smoothing algorithm removes spurious motion vectors that does not follow prior model, in this case inter-scale-Markov-random-fields modeling. The result is not really smooth, but it already reduce the result of adaptive matching.

In this presentation, the purpose is to introduce a new way to do the smoothing. To produce a really good "true" motion fields, several preparation must be done, e.g. filtering the image, reducing the size of image or the use of region based matching. The 4 by 4 block matching result is shown. In fig. 8 (f), a little bit modification of the Dubois-Konrad algorithm is used, ${ }^{4}$ this MRF modeling algorithm uses iterative smoothing and line fields. If the result of (d) is inserted in this algorithm, the result has higher entropy, it shows that the global solution has been contributed by multiscale smoothing. Fig. 8 (h) shows the result of 4 by 4 block matching, where the multiscale smoothing influences the result.

\section{CONCLUSION}

The separation between matching stage and smoothing stage for motion estimation is introduced. The matching stage that employs implicit smoothness is block matching. The adaptive block size matching in this paper is the matching technique thas has lowest smoothness and highly match result, althought it should no be a good "true" motion vectors. The multiscale smoothing as a quadtree based modeling for the motion vector is used here to smooth the motion vectors without any computation that relates to intensity pixels. The possibilities to give the information for the multiscale smoothing algorithm such as the measurement error from matching algorithm exist in this framework. Even "no-measurement" information is a good information for mulstiscale smoothing algorithm to merge the motion vector for those sites from the multiscale neighborhood vectors. This type of strategy can be implemented for block matching, dense motion field and optical flow result. It can be tried to region based matching for further studies.

\section{REFERENCES}

1. L. Keng-Pang, C. Man-Nang, and A. Das, "Dense motion field and uncovered region estimation for low bit-rate video coding application," in Visual Communications and Image Processing '99, Y.-Q. Z. Kiyoharu Aizawa, Robert L. Stevenson, ed., Proc. SPIE 3653, pp. 547-567, 1999.

2. J. Konrad, "Motion detection and estimation," in Image and Video Processing Handbook, A. Bovik, ed., p. chap 3.8, Academic Press, 1999. 


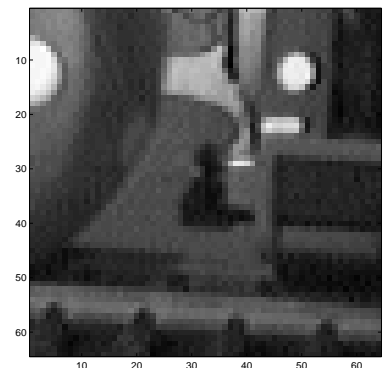

(a) Calendar train 1

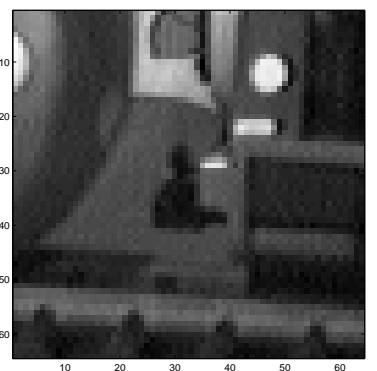

(b) Calendar train 2

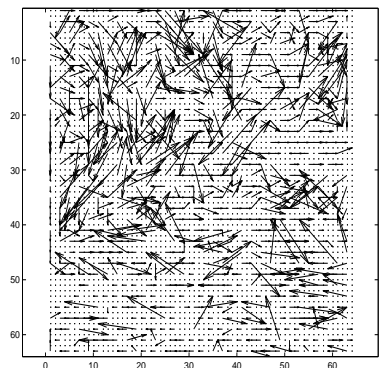

(c) Adaptive matching result

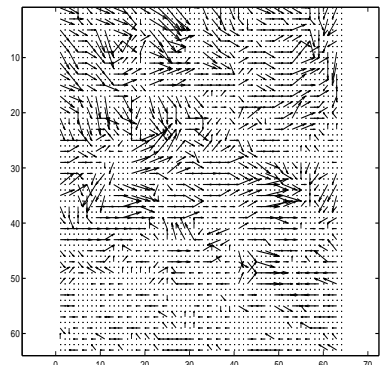

(d) Adaptive BM and multiscale smoothing

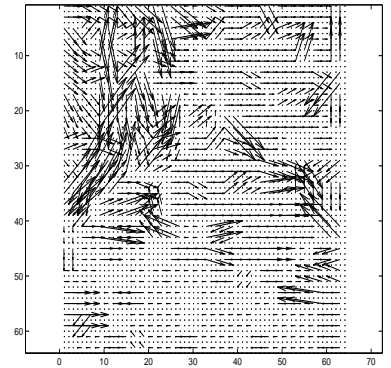

(e) 4 by 4 block
matching

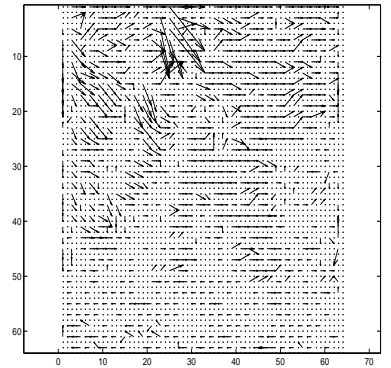

(f) Iterative method

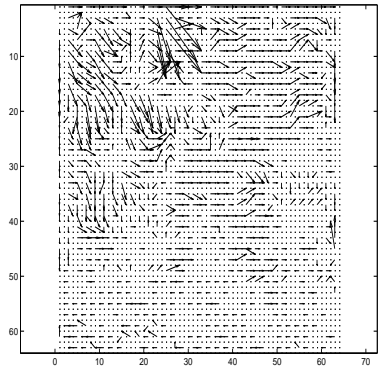

(g) Multiscale
smoothing and

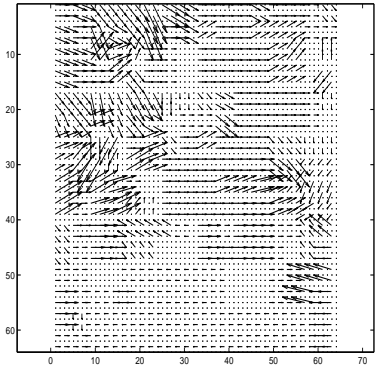

(h) 4 by 4 block matching - multiscale smoothing

Figure 8. Adaptive matching and multiscale smoothing

3. M. J. Black, Robust Incremental Optical Flow, Yale University, Ph. D. Thesis, New Haven, 1992.

4. J. Konrad and E. Dubois, "Bayesian estimation of motion vector fields," IEEE Trans. Pattern Analysis and Machine Intelligent, vol. 14, pp. 910-927, 1992.

5. W. Li and E. Salari, "Successive elimination algorithm," IEEE Trans. Image Processing, vol. 4, pp. 105-107, 1995.

6. H.-S. Wang and R. M. Mersereau, "Fast algorithms for the estimation of motion vectors," IEEE Trans. Image Processing, vol. 8, pp. 435-438, 1999.

7. M. Brunig and W. Niehsen, "Fast full search block matching based on combined sad and mse measures," in Visual Communications and Image Processing '99, Y.-Q. Z. Kiyoharu Aizawa, Robert L. Stevenson, ed., Proc. SPIE 3653, pp. 439-449, 1999.

8. S. Suryadarma, C. Man-Nang, and W. Kin-Keong, "Adaptive blocksize observation model for mrf modeling of motion field," Proc. ICICS, vol. 2, pp. CD-ROM, 1999.

9. K. C. Chou, A. S. Willsky, and A. Benveniste, "Multiscale recursive estimation, data fusion, and regularization," IEEE Trans. Automatic Control, vol. 39, pp. 464-478, 1994.

10. M. R. Luettgen, W. C. Karl, and A. S. Willsky, "Efficient multiscale regularization with applications to the computation of optical flow," IEEE Trans. Image Processing, vol. 3, pp. 41-64, 1994.

11. S. Kalra and C. Man-Nang, "Bidirectional motion estimation via vector propagation," IEEE Trans. Circuits and Systems for Video Technology, vol. 8, pp. 976-987, 1998.

12. T. T.-J. Ho, Multiscale Modeling and Estimation of Large-Scale Dynamic Systems, Massachusetts Insitute of Technology, http://ssg.mit.edu/group/hot, Ph. D. Thesis, Cambridge, 1998. 
13. A. M. Tekalp, Digital Video Processing, Prentice Hall, Upper Saddle River, 1995. 